\title{
Estigma y auge de prestigio: El cambio $f>h$ en caste- llano y gascón visto desde la sociolingüística histórica y la lingüística variacional ${ }^{1}$
}

\author{
Frank JODL \\ Universidad de Siegen \\ jodl@romanistik.uni-siegen.de
}

\section{RESUMEN}

En este artículo, me gustaría volver al problema del origen de un determinado fenómeno del cambio lingüístico, es decir, [f] -> [h] - en castellano y gascón, al contrastar los factores estructurales (internos) con los sociales (externos). En el curso de mi argumentación, también me dirijo a las ventajas de una teoría que combina factores internos y externos (Tuten 2003), finalmente se reafirma, sin embargo, el papel decisivo que desempeñan los factores sociales.

Palabras clave: cambio lingüístico, factores internos contra factores externos, la aspiración de f- en castellano y gascón.

[Recibido, mayo 2014; aprobado, diciembre 2014]

Stigma and increased prestige: The change $\mathrm{f}>\mathrm{h}$ in Castilian and Gascon seen from the perspective of historical sociolinguistics and variational linguistics

\begin{abstract}
In this article, I revisit the problem of the origin of a certain language change phenomenon, i.e. [f]- > [h]- in Castilian and Gascon, by contrasting structural (internal) factors with social (external) ones. In the course of my argumentation, I also address the advantages of a theory that combines internal and external factors (Tuten 2003), finally reaffirming, however, the decisive role played by social factors.
\end{abstract}

Keywords: language change, internal versus external factors, aspiration of $\mathrm{f}$ - in Castilian and Gascon.

\footnotetext{
${ }^{1}$ Quisiera expresarles mi más sincero agradecimiento a la Dra. Laura Ramirez Sainz, Siegen, y al Dr. Miguel García García, Barcelona, por la ayuda que me han brindado durante el proceso de la redacción de la versión española del presente trabajo. Cualquier error que aún contenga corresponde - obviamente - a mi responsabilidad.
} 


\section{Introducción}

\subsection{Generalidades}

Evaluando las argumentaciones vertidas en un número considerable de los artículos más recientes que han sido publicados con el objetivo de aclarar la esencia ${ }^{2}$ del cambio lingüístico, nos llama la atención el hecho de que, al punto de vista estructural, que no precisa factores externos o sociales para explicar dicho fenómeno, ya no se le atribuya una posición central, cf. Argente (1998: 9):

Factores estructurales, en suma, determinarán tal vez cuáles sean los cambios posibles y los cambios previsibles, pero poco aportarán acerca del proceso histórico concreto que haya conducido a ellos.

Penny (2009: 54) destaca de manera aún más explícita que son los factores sociales, y no los estructurales, los que desempeñan el papel decisivo en el cambio lingüístico:

La lengua está en un constante proceso de recreación, reconstruida a cada paso por las comunidades cambiantes que la emplean. La sociolingüística nos ha enseñado a los historiadores de la lengua que es insuficiente decir que la lengua cambia. Lo que ocurre es que los hablantes cambian la lengua.

En este marco cabe citar otro trabajo de Penny, en el que afirma que el factor sociolingüístico asume una posición primordial, cf. Penny (1972: 473):

Nevertheless, the difference between [ $\phi w e ́ r t e]$ and [hwérte] is probably not so much a geographical one as a social one, [ $\phi$ wérte] being regarded as 'correct' and [hwérte] as 'rustic'.

Sigue el mismo autor diciendo que, cf. Penny (1972: 479):

The conquest of America took place at a time when popular/rural Castilian employed both the sets of allophones [...], according to area. Both these types occur widely in the popular speech of Spanish America [...], although subject to the minor modifications to be detailed in $\S 13$. Naturally, the middle and upper classes of American society followed the urban Castilian norm [...].

Teniendo en cuenta las nociones relativas al cambio lingüístico preconizadas por neogramáticos y estructuralistas - que partieron del principio de que los cambios lingüísticos se caracterizan por estar sujetos a un cierto automatismo ${ }^{3}$, también colectivo por lo que concierne a los hablantes - es intersante observar como Koch (2005) plantea el problema centrándose en el individuo bien preciso que causó una cierta innovación lingüística y que parece corresponderse al "paciente cero" de la medicina: ¿Quién forjó la innovación? cf. Koch (2005: 236) $)^{4}$ :

\footnotetext{
${ }^{2}$ Cf. resumen en Jodl (2013c, en prep.).

${ }^{3}$ Piénsese en la teoría de la "invisible hand" según Keller (1994), criticada en Kabatek (2005). Parece que el problema nace de una insuficiencia en cuanto a la distinción de 'innovación” versus 'cambio', cf. Coseriu (1974: 68). Cf. también Jodl (2008) y Jodl (2013c, en prep.). Es también interesante la indicación anti-estructuralista que nos da Penny (1972: 465) que destaca los puntos débiles de la opinión de Martinet (1951-52: 137).

${ }^{4}$ En Jodl (2013c, en prep.) hicimos hincapié en que es importante considerar el cambio lingüístico como fenómeno heterogéneo que describe los diversos tipos de cambio. En cuanto al ejemplo que damos aquí no es preciso entrar en esta tipología del cambio lingüístico, basta con la primera distinción primordial que consta de "innovación" versus “adopción/cambio", cf. Coseriu (1974: 68).
} 
In dem Augenblick, wo erstmals ein Sprecher travailler, 'sich abmühen' innovierend für 'arbeiten' verwendet, bedient er sich einer expressiv-hyperbolischen Metonymie.“

[Traducción F.J.: En el momento en que un locutor utiliza por primera vez 'travailler' (literalmente: torturarse trabajando/afanarse) de modo innovador para expresar el concepto de 'obrar/trabajar', éste se sirve de una metonimia expresiva-hiperbólica."]

Es aquí donde, una vez más, nos damos cuenta de la importancia que la lingüística moderna atribuye a hechos sociales. La investigación de tales detalles, consecuentemente, es crucial, si tenemos presente que un número elevado o un número mínimo de individuos hablantes portadores de una innovación lingüística ha sido considerado decisivo ${ }^{5}$ para que una innovación lingüística pueda imponerse y convertirse en un "cambio". Que esto no es necesariamente así lo demuestran Koch, al que acabamos de citar, y además Bailey/Maroldt (1977: 28, a propósito de la cuestión de cómo el francés antiguo influenció el inglés antiguo, énfasis en letra negrita F.J.):

\begin{abstract}
More important than this parade of numbers [Berndt's requisito de un número bastante alto de individuos expuestos al contacto lingüístico, F.J.], however, seems to be the social status of the immigrants and their language. Berndt is probably right in assuming that the highest nobility [...], whose interests were most intensively engaged in the Continent and who spent most of their time in France, were exclusively French in orientation. This, however, was probably not true of the 6500-7200 knights, the lesser feudal representatives all over the country who were subject to language contact. Intermarriage, English nurses and playmates for the children, and the necessities of everyday life and administration must have caused intensive language contact.
\end{abstract}

Todo esto no quiere decir que se haya dejado de reconocer que también los factores internos / estructurales están vinculados con el fenómeno del cambio lingüístico ya que sí engendran innovaciones lingüísticas. Este papel de los factores internos, sin embargo, se restringe cum grano salis al ámbito fonético-fonológico y - como acabamos de decir - al estadio de la innovación: Será el próximo estadio, el que decide si una innovación lingüística se impone y así - de innvoación - se convierte en verdadero cambio, lo que vuelve a llevarnos al ámbito de los hechos sociales y extra-lingüísticos, cf. Jodl (2013c, en prep.). Antes de profundizar y concretar tales asuntos quisiéramos dar las siguientes indicaciones respecto a la estructura del presente artículo:

1. Introducción

2. La aportación de la lingüística estructural para explicar el origen de la innovación f- $>$ h- y puntos débiles

3. La aportación de la sociolingüística histórica

4. Conclusiones

Considerando el papel crucial que desempeña la koineización en el marco de los procesos que quisiéramos detallar en el presente trabajo, cabe explicar cómo proponemos percibir este fenómeno lingüístico, antes de pasar a otros detalles. Según describen Selig (2008) y Grübl $(2010)^{6}$ en sus resúmenes acerca del término "koineización", su uso es todo menos

\footnotetext{
${ }^{5}$ Cf. Holtus/Kramer (2010: 154), Berndt (1965=1969), citado en el párrafo 3.1. (abajo), Ineichen (1997: 11). Habrá que volver a este asunto, más abajo. Cf. también Jodl (2004).

${ }^{6}$ Véanse también las indicaciones de Tuten (2003) en las que se apoya, entre otras, Grübl (2010).
} 
unitario. Prescindiendo de dos adaptaciones que vamos a aportar, seguimos aquí la definición de Trudgill (1986: 107s.):

A historically mixed but synchronically stable dialect which contains elements from the different dialects that went into the mixture, as well as interdialect forms that were present in none.

La primera adaptación consta de una precisión que se basa en Tuten (2003), trabajo que demuestra que en nuestra zona concreta nos encontramos frente a un tipo de koineización que se caracteriza por el hecho de que las variedades que forman la koiné contribuyeron a ella de una manera equilibrada, en concreto: no hubo variedad que hubiera desempeñado un papel primordial. ${ }^{7}$ En cuanto a la segunda adaptación, quisiéramos señalar que ha habido una discusión muy intensa en cuanto al papel del factor de la "identidad"8 durante el proceso de la koineización: Seguimos la concepción de Tuten (2008) según la cual la "identity" es un requisito de la koineización y no una de sus consecuencias ${ }^{9}$.

\subsection{Objetivo del presente artículo:}

Sirviéndose de argumentos suplementarios ${ }^{10}$ respecto a los vertidos hasta ahora, el presente artículo tiene el objetivo de demostrar - que:

a.) es posible explicar la innovación y el cambio $\mathrm{f}->\mathrm{h}$ - restringiéndose al factor del contacto lingüístico con el vascuence (revalorización de la posición de Ramón Menéndez Pidal) aunque

\footnotetext{
${ }^{7}$ Selig (2008: 76) propone una tipologia de situaciones de koineización que tiene en cuenta las distintas constelaciones históricas que crean situaciones que a priori se han denomindado "koineización". En nuestro caso, tiene que ver - según Selig, ibd., y de acuerdo con Tuten (2003) - con una "koineización” en el sentido más estricto, es decir: con una "immigrant koiné" nacida de un proceso durante el cual todas las variedades lingüísticas que contribuyeron a la koiné tuvieron el mismo estatus social y contribuyeron de manera equilibrada. Este tipo se distingue, por ejemplo, del sub-tipo del "dialect mixing", que puede ser caracterizado como situación en la que una de las variedades que contribuyen a la koiné desempeña un papel primordial.

${ }^{8} \mathrm{Cf}$. el breve resumen de la discusión y la bibliografía respecto a la discusión en Jodl (2013c, en prep.).

${ }^{9}$ La convicción de que la "identity" es el producto de y no el requisito para la koineización se remonta a Trudgill $(2008 \mathrm{a}+\mathrm{b})$.

${ }^{10}$ Por lo que concierne a las opiniones proferidas a propósito de nuestro tema (véase también el resumen dado en Jodl 2013c, en prep.), en el presente artículo tan solo consideramos los trabajos acogidos en la sintésis de Tuten (2003: 132-136), quiere decir que no consideramos aquí el debate llevado por Anthony Naro y José Luis Rivarola en la "Zeitschrift für romanische Philologie" (1972), vol. 88, cuaderno 1/3 y que nos restringimos a los trabajos siguientes, de modo ejemplar:

a.) Menéndez Pidal (hasta 1980): Esgrime exclusivamente argumentos externos a la lengua: f- $>$ h- es exclusivamente un fenómeno de contacto vascuence-romance

b.) Trask (1997): f- > h- no tiene nada que ver con el contacto vascuence-romance, la innovación en cuestión está debida a tan sólo factores internos a la lengua es decir a factores estructurales

c.) Tuten (2003: 133-136): Combina los factores internos y externos, cf. también Jodl (2000), (2004) y (2005) a propósito de una situación análoga.

d.) Penny (1972): Es muy probable que la innovación $\mathrm{f}>\mathrm{h}$ tenga causas únicamente internas a la lengua, PERO: en cuanto a la evolución particular de f- > h- ante [w], [r] y [1], como en p. ej. "fuerza" en vez de "huerza", sí entra en juego el contacto lingüístico, en este caso concreto el del superestrato/adstrato franco/occitánicorromance que causaría la restitución de f- en ciertos casos.
} 
b.) un planteamento policausal tendría que tener la mayor plausibilidad (posición de Tuten 2003, véase también Jodl 2000 y 2004, 2005 y 2013a)

c.) hay que atribuirle más peso al factor extralingüístico de "prestigio"

d.) surgen problemas insuperables si nos limitamos a los factores estructurales, véanse las citaciones Argente (1998: 9) y Penny (2009: 54), más arriba.

Aproximándonos a una propuesta para la solución del problema de cómo explicar el fenómeno $\mathrm{f}->\mathrm{h}-$, nos centraremos en el deficit siguiente:

los trabajos estrictamente estructuralistas no respetan lo suficiente la necesidad de distinguir entre "innovación lingüística" y "adopción de la innovación = verdadero cambio"11. Así consideramos oportuno llevar al primer plano los pasos siguientes: averiguar los factores que pueden causar una innovación, sea interna o externa al sistema, para luego pasar al aspecto variacional, quiere decir la investigación de la co-existencia de variantes y los procesos de elección, para integrar los resultados a la discusión acerca del origen de los relativos cambios.

\section{El punto de vista estructural}

\subsection{Puntos débiles del punto de vista estructural para explicar el origen de la innova- ción $\mathbf{f}->\mathbf{h}^{-12}$}

Como dijimos más arriba, nos centraremos en la distinción “innovación lingüística” versus "adopción/generalización de la innovación = cambio lingüístico" lo que nos lleva a tratar primero la cuestión de cómo pudo nacer la innovación f- $>$ h-.

Ya en el nivel de la innovación hay 4 argumentos que Trask (1997: 424-429) esgrimió contra la tésis 'pro contacto' defendida por Menéndez Pidal. Ampliando la argumentacion presentada en Jodl (2013c, en prep.), quisieramos replicar estos argumentos traskianos ${ }^{13}$ :

\section{Argumento a.):}

Resumen ${ }^{14}$ de la opinión de Trask (ibd.): Si f- $>$ h- del castellano se remontase a una interferencia con el vascuence, el fenomeno tendría que ocurrir en cualquier posición, como en el caso del gascón. Sin embargo, en castellano la innovación no se produjo ante [w] como en "fuerza" y ante líquidas [r], [1] como en "frío" contrariamente al gascón, donde se produjo f- $>$ h- incluso ante [w] y [r], [1] como demuestran huelha de FOLIA, hrét de FRIGIDU y hlor de FLOR, véase más abajo, párrafo 2.3.

Nuestra objeción sería la siguiente: Los diagnósticos dialectal y diacrónico conseguidos por Menéndez Pidal (1980: 198-232), Penny (1972) y además Rosales Solís (2010), Navarro

\footnotetext{
${ }^{11}$ Cf. Coseriu (1974: 68) y Jodl (2013c, en prep.).

${ }^{12}$ En cuanto a la precocidad de la innovación, cf. Ramos Remdios (2000: 50): La primera atestación puede remontarse al año 864, véanse los cartularios de Valpuesta. En cuanto a problemas de datación, cf. Jodl (2016, 95).

${ }^{13}$ Cf. también el resumen en Tuten (2003: 133ss.). A algunas replica Tuten, nosotros intentaremos aquí aducir ulteriores argumentos contra el punto de vista estructural, cf. también Jodl (2013c, en prep.).

${ }^{14}$ Cf. Tuten (2003: 133).
} 
(1974), Flores (1983) y Rueda Chaves/Navas Escorcia (2011: 60, nota 6) demuestran que f$>$ h- puede haber ocurrido también ante [w], [r] y [1], así que:

f- > h- se produjo también ante sonidos líquidos: cf. p. ej. Penny (1972: 470):

\author{
ante [1]: FLACCIDU > lacio, FLAMMULA > lambra \\ ante $[\mathbf{r}]$ :
}

Es interesante ver que en ciertas regiones de Hispanoamérica hallamos formas como "hruto" [hruto] y "hrío” [hrio] en vez de „fruto“ y "frio, cf. Navarro (1974), Flores (1983) y Rueda Chaves/Navas Escorcia (2011: 60, nota 6). ${ }^{15}$ Estas formas podrían ser consideradas como arcaísmos del castellano así que sí sería posibile esgrimir la opinión de que f- $>$ h- en el castellano antiguo se produjo también ante [r], como en gascón ${ }^{16}$.

Además, f- $>$ h- se produjo también

ante [w]: Menéndez Pidal (1980: $200+221$ ):

Variantes dialectales peninsulares ${ }^{17}$ e hispanoamericanas, como "huego", "huerte" en vez de "fuego" y "fuerte", etc., véanse también ciertas variedades del español judío "huersa" en vez de "fuerza" ron restricciones, p. ej. ante $[\mathrm{w}]$, para la innovación $/ \mathrm{f} />/ \mathrm{h} /$. Esta opinión podría ser apoyada por el hecho de que en el español de p. ej. Nicaragua /f/ se articule como $[\phi]$ o incluso como [h] también ante [w], cf. Quilis (1995: 382) y Rosales Solís (2010: 143). Nos resulta plausible ver en este fenómeno un arcaísmo del castellano medieval pensinsular, ${ }^{19} \mathrm{cf}$. también Penny (1972, 465f. + 470f.).

Sin embargo, interpretando a Penny (1972: 473s., 479s. y 482), es posible que también en este caso se produzca un problema de cronología respecto al nacimiento de [f] $>$ [h] ante

\footnotetext{
${ }^{15}$ Cf. a propósito del español nicaragüense el resumen en Jodl (2013b: 607). Como dijimos también arriba, la existencia de $[\phi r]$ se puede interpretar como residuo de una variante fonética medieval que pudo convertirse en [hr]. ${ }^{16}$ Esto podría coincidir con Penny $(1972: 470)$ que constata: “[...] that Castilian levels the allophones $[\phi]$ and $[\mathrm{h}]$ of $[\phi]$ in favour of $[\mathrm{h}] \ldots$.... [lo refiere también a los casos de $[\phi \mathrm{r}]$ ]". Pero podríamos encontrarnos ante un problema de cronología: Según dice Penny (1972: 474s.), [hr] sería una variante, o sea, el resultado de [xr] así que [hr] no podría ser un fenómeno medieval lo que esgrimimos en la presente argumentación. Dado que el punto de vista estructural parte del principio de que la evolución $\mathrm{f}->\mathrm{h}$ - no fue causada por factores como el contacto lingüístico, tenemos que presuponer que también en gascón, donde sí tenemos [hr] (sin necesidad de [xr]) el alófono [ $\phi \mathrm{r}]$ pudo llegar a [hr] de manera "natural" o "automática" aunque, como dice Penny (1972: 467) en la posición ante [r] el alófono [ $\phi]$ es menos propenso a convertirse en [h]. Así nos permitimos hacer la pregunta de si a lo mejor nos encontramos frente al proceso inverso: [xr] nació de [hr]. Así que quizá quepa efectuar un re-analisis o una re-interpretación de las formas dialectales hispanoamericanas, véase arriba.

${ }^{17}$ Cf. Ménendez Pidal (1980), p. 200+221, explicación general; p. 214: "huéya” de FOLIA en Oseja, Asturias orientales; p. 229: "el habla villanesca toledana conservó por algún tiempo la aspiración hasta en huerte y hue [...].” p. 230: Salamanca: "[...] pues los pastores de las aldeas de Ledesma y de Salmanca, a fines del siglo XV, ya usaban corrientemente la $h$, hasta en huera, huerza, hué, y muy rara vez recordaban la $f[. .$.$] ."$

${ }^{18}$ Cf. Nezirovic (2002: 111) y también Lapesa (1981/2008: 442).

${ }^{19}$ Ulterior confirmación nos fornece Rosales Solis (2010, 143, nota 7), apoyándose en Quesada Pacheco (2000 [sic!], 73 [correcto: 2002/2008, 73]). Hace hincapié en las hipercorrecciones del tipo [fuy'ar] statt [xuy'ar] etc. Estas hipercorrecciones son muy comunes en el español de las Américas lo que habla a favor de un arcaísmo peninsular, como dijimos arriba.
} 
[we]: En cuanto a la argumentación vertida en el presente artículo, [f] $>$ [h] ante [we] tiene que ser una evolución medieval, lo que no se puede confirmar sin problema si tenemos en cuenta la argumentación de Penny (ibd.) según la que [hwe] tan sólo pudo producirse después de que $[\chi]$ se hubiera originado de [3] y [S] a partir del siglo XVI (Penny 1972: 479 s.), aproximadamente. En este caso, [hw $]$ sería una variante de $[\chi w \varepsilon]$ y consecuentemente una forma muy tardía. ${ }^{20}$ ¿Pero por qué $[h \phi \varepsilon]$ no debería haber pasado a [hwe], dando lugar, más tarde, a $[\chi w \varepsilon]$ ? Es en Penny (1972: 480s.) dónde encontramos una pista para poder esclarecer esta pregunta:

It is also necessary to account for the late orthografical appearance of $\mathrm{h}$ before [w], if, as I have argued, the allophone $[h \phi]$ was used in this position from the earliest times down to the modern period, at least in rural Castilian. [...]. The sudden and late appearance of such spellings is to be explained in the following way. While both urban and rural Castilian used [h $\phi]$ before [w], the spelling f was entirely appropriate to both [...]. But once standard castilian [h $\phi]$ had been replaced by [f], surviving rural [h $\phi]$ was reasonably felt to have more in common ${ }^{21}$ with $[\mathrm{h}]$ (increasingly spellt $\mathrm{h}$ ) than with standard [f] (written $f$ ).

Además, tendríamos que preguntarnos si no nos encontramos ante el caso contrario, es decir: $[h w \varepsilon]$ nació primero así que $[\chi w \varepsilon]$ sería una variante de [hwe], cf. en general Noll (2009: 82): las primeras apariciones en documentos escritos no prueban nada a propósito de la aparición en el lenguaje hablado:

Faktisch stellt der prozentuale Anteil an Sprechern aus einer Region jedoch genauso wenig einen Beweis für sprachliche Beeinflussung dar wie die Chronologie von Sprachbelegen. [Efectivamente, ni el porcentaje de hablantes de cierta región ni la cronología de comprobantes lingüísticos representa una prueba para una influenciación lingüística. (Traducción F.J.)].

En este sentido tendrían más peso los dialectalismos asturianos, como "Central Asturian [hwérte], [hwénte]" 22 porque en estos casos se puede descartar la duda de si [h] en [hwe] es muy tardío (por haber nacido de $[\chi w \varepsilon]$ ) y esto debido al hecho de que $[\chi]$ no ha sido un sonido asturiano.

Sean cuales fueren los problemas de la primera documentación escrita, ${ }^{23}$ según lo expuesto más arriba parece posible que en el lenguaje espontáneo de los hablantes de iberorromance (septentrional) - en un primer momento ${ }^{24}$ - /f/ se convirtiera en $/ \mathrm{h} /$ también ante /1/, /r/ y /w/, como en gascón, y que así, desde el punto de vista diacrónico, no hubiera diferencia entre el castellano y el gascón. El hecho de que /h/ precisamente en estas posiciones no haya sobrevivido - lo que parece concerner sobre todo a la variedad estándar del castellano - es una evolución secundaria debida al contacto con variedades galorománicas, cf. Penny (1972: 476ss. y 482).

\footnotetext{
${ }^{20}$ En las páginas 480f., Penny (1972) explica por qué las grafías $<$ huerte $>$, $<$ huera $>,<$ huerza $>,<$ huego $>$ aparecen bastante tarde (siglo XV, eventualmente XIV).

${ }^{21}$ Esto habla en favor de la hipótesis de la koineización como factor respecto a la evolución $\mathrm{f}>\mathrm{h}$ en general.

${ }^{22}$ Cf. Penny (1972: 481). Véanse también (ibd.) las páginas 469 y 473.

${ }^{23} \mathrm{Cf}$. nota 20 , arriba.

${ }^{24} \mathrm{Cf}$. Tuten (2003: 136) cuando habla de la "overgeneralization of the [h] allophone" que puede haber ocurrido durante la "Burgos-phase koineization among native romance speakers, particularly since it is a more natural change".
} 
Incluyendo de esta manera el punto de vista variacional, se ve que es problemático alegar que el carácter del fenómeno f- $>$ h- del castellano antiguo se opone al del gascón: En el primer estadio de la innovación $\mathrm{f}$ - $>$ h- puede haber sido generalizado como ocurrió en gascón y el factor "contacto con el vascuence" no puede ser desdeñado con el argumento de una presunta distribución irregular de $\mathrm{f}>\mathrm{h}$ en el proto-castellano así que no se podrá justificar fácilmente una independencia recíproca del fenómeno castellano y del gascón. Recordamos al respecto, una vez más, el principio coseriano de hacer hincapié en la necesidad metodológica de distinguir entre "innovación" versus "cambio".

\section{Argumento b.):}

La [f] inicial ha sido adoptada por los vascohablantes de distintas maneras: tenemos ejemplos de préstamos que - en vez de [h] inicial - acusan [p] o [b] iniciales, véase $\mathrm{p}$. ej. lat. FESTA $>$ vasc. pesta $^{25}$. Este hecho podría contradecir la teoria según la cual el cambio $\mathrm{f}->$ hen protocastellano se debería a una interferencia de los vascohablantes. Ahora bien, también este argumento ha encontrado respuesta: Es otra vez Tuten (2003: 134) el que nos recuerda que las sustituciones fonéticas que ocurren en el marco de los préstamos léxicos tomados por monolingues no están sujetos a los mismos mecanismos de adopción que los fenómenos de sustitución fonética que ocurren en el marco del language shift cuyo primer estadio es el bilingüismo, para luego llegar al abandono de la lengua autóctona o sea al language shift que engendra los fenómenos de sustrato ${ }^{26}$.

\section{Argumento c.):}

Respecto a la Navarra y el Aragón proto-medievales, también se tiene que contar con una influencia vasca considerable, sin embargo, hoy día, en navarro-aragonés no encontramos el rasgo f- $>$ h-. Hemos propuesto primeras pistas en cuanto a posibilidades de réplica en Jodl (2013c, en prep.) señalando que [h]-, de lat. [f]-, sí puede haber existido también en navarro-aragonés, como variante fonética/alofónica, a nivel de innovación y que la pregunta de por qué la innovación no se ha impuesto en navarro-aragonés es otro asunto. Lo que quisiéramos añadir aquí es que incluso hay testimonios, de índole toponomástica, que demuestran que el punto de vista variacionista que esgrimimos en Jodl (2013c, en prep.) no necesariamente tiene que quedar en un plan teórico:

Menéndez Pidal (1980: 211f. + 220) y Penny (1972: 467) nos dan algunos ejemplos del navarro-aragonés que podrían confirmar que $\mathrm{f}-\mathrm{>}$ h- también se produjo en navarro-aragonés, cf. p. ej. Menéndez Pidal (1980: 212), topónimo, cerca de Huesca, Aragón:

FAUCE > Oçe (1095);

FAUCE > Oze (1099) el actual "Hoz de Barbastro"

Otra confirmación se encuentra en Tuten (2003: 134) que apunta a la situación sociolingüística: En Navarra - contrariamente a Castilla - no hubo una situación de koineización lo

\footnotetext{
${ }^{25}$ Cf. para un resumen Tuten (2003: 134) o Menéndez Pidal (1980: 217).

${ }^{26}$ Penny (1972: 466) nos da una explicación fonológico-combinatórica a través del alófono [ $\left.\phi\right]$.
} 
que dio por resultado que el romance hablado en Navarra fue mucho más homogéneo ${ }^{27}$ que en Castilla así que los individuos vascos que aprendían el romance tenían un "more stable and homogeneous input", cf. Tuten (2003: 134):

When Basque [...] learners were presented with clear and consistent models, used by a majority of speakers in the target community [romance, F.J.], it is probable that they (or their children) acquired the target forms correctly.

A esto se suma, cf. Tuten (2003: 134):

[....], the relative demographic stabilty of Navarre would have contributed to much stricter norm enforcement, which would have served to suppress Basque interlanguage features not shared by native Romance speakers [...].

Como dijimos en Jodl (2013c, en prep.) tendríamos que preguntarnos adémas cuál era el estatus social de los individuos vascos en la sociedad proto-castellana y la proto-navarra si tenemos presente que el éxito de la innovación $\mathrm{f}->\mathrm{h}$ - en castellano obedece al principio al que proponemos llamar "un error se convierte en norma".

\section{Argumento d.):}

El contacto con el vasco no puede haber desempeñado un papel decisivo ya que hay zonas en Italia en las que la innovación $\mathrm{f}>\mathrm{h}$ ocurrió también - obviamente sin que existiera allí el contacto con el vasco (Trask 1997). Esgrimiendo que se tendría que tener en cuenta el siguiente contra-argumento suplementario en lo referente a la posición de Trask vamos más allá que Tuten (2003), cf. Jodl (2013c, en prep.): El fenómeno de la existencia de una /1/ velar en el español regional de Cataluña se atribuye - sin duda - al contacto con el catalán. El hecho de que la /1/ velar exista también en inglés o portugués etc. - sin que haya, en los territorios en que se hablan estas lenguas, un contacto histórico con el catalán - nunca ha inducido a nadie a poner en duda la opinión según la cual el origen de la /1/ velar en el español regional de Cataluña se debe al contacto con la lengua catalana. Este ejemplo demuestra que la argumentación de Trask no puede ser generalizada para después ser aplicada al ejemplo concreto del cambio [f-] $>$ [h-], cf. argumentos suplementarios en Menéndez Pidal (1980: 218s.).

\subsection{Resultado intermedio}

La tesis según la cual innovación y cambio f- $>$ h- se remontarían a una interferencia vasca tal como la forjó Ramón Menéndez Pidal no carece de fundamento:

$\rightarrow \mathrm{f}->\mathrm{h}$ - no es el único fenómeno explicable por interferencia vasca que comparten el gascón y el castellano, véase la a- protética ante $[\mathrm{r}]$ inicial: castellano: arrepentir, gascón: arròda etc., cf. Bertoldi (1931) y Menéndez Pidal (1980: 207)

\footnotetext{
${ }^{27}$ Tuten (2003: 134): "We know that [...] the degree of demographic and dialect mixing was far less in there than in Castile."
} 
Resultado intermedio: El diagnóstico diacrónico, dialectológico y variacional muestra que la innovación $\mathrm{f}->\mathrm{h}$ - del gascón y la del proto-castellano al principio no se distinguieron respecto a la distribución del fenómeno. Pero sigamos ocupándonos de los argumentos de la teoría anti-contacto.

\subsection{La aportación del punto de vista estructural para explicar el origen de la innova- ción $\mathbf{f}->$ h-}

El punto de vista estructural se basa en la argumentación, cf. Penny (1972: 465), de que - durante la antigüedad - en el latín vulgar y el protorromance de la totalidad de la zona septentrional de la Iberorromania no existía el fonema /f/ como [f], en concreto como sonido fricativo labio-dental. ${ }^{28}$ En consecuencia, el alófono $[\phi]$ - que no es nada más que uno de los posibles alófonos del fonéma /f/ del latín - en el latín vulgar del norte de la península ibérica se convertiría en el fonéma fricativo bilabial $/ \phi /$ que - a su vez - se compone de los alófonos siguientes, cf. Penny (1972: 467):

1.) alófono $[\mathrm{h}]$ (procedente de $[\phi])$ :

ante /o/ y /u/ sílbicos: FORNU

$$
>\text { ['hornu }]
$$

2.) alófono $[\phi]$

ante las demás vocales:

ylas líquidas /r/ y /1/

FARINA $\quad>$ [фar'ina $]$

$\mathrm{y}$ ante $/ \mathrm{j} /$

FRIGDU $>$ ['申riu]

FJERRU $\quad>$ ['фjer:u]

3.) alófono $[M]=[h \phi]$ (nacido por relajamiento de $[\phi w]$ ante $[w]$ ):

ante $/ \mathrm{w} / \quad$ FUERZA $>\left[M w^{\prime} \varepsilon r t s a\right]=\left[h \phi^{\prime} \varepsilon r t s a\right]$

El alófono $[\phi]$ desempeña un papel decisivo para el planteamento estructuralista estricto que niega la relevancia de factores externos respecto al cambio lingüístico, cf. Tuten (2003: 135), según Penny (1972: 466f.) $)^{29}$ :

Given that $[\phi]$ is lax and inherently unstable $[\ldots]$, an easy and probably automatic solution in fast speech would have been to simply relax the articulation of the lips even further, which would lead to the articulation (and/or perception) of a silent vowel similar to a glottal aspirate.

Dadas estas características del sonido [ $\phi]$, éste pudo convertirse en [h], SIN que contribuyeran factores EXTERNOS como el contacto lingüístico con el vascuence. Esta evolución dio origen a la siguiente distribución del alófono $[\mathrm{h}]$ procedente de $/ \phi /$, cf. Penny (1972: 466f.):

Ante $[\mathrm{o}]$ y $[\mathrm{u}]$ era particularmente fácil que $[\phi]$ se convirtiera en $[\mathrm{h}]$ :

FORNU $=[$ '申ornu $] \quad>$ ['hornu $]$

para luego expandirse a las posiciones ante: [a], [e], [i] y [j]:

\footnotetext{
${ }^{28}$ Véase también el resumen en Tuten (2003: 132-136). Del resto, Wartburg (1928: 459) también partió del principio de que existió un alófono bilabial de /f/, pero restringió su existencia a la zona colindante con el dominio vasco.

${ }^{29}$ Cf. también Wartburg (1928: 459).
} 
$\begin{array}{ll}\text { FARINA } & >\text { harina } \\ \text { FOETERE/FEDER } & >\text { heder } \\ \text { FILIU } & >\text { hijo } \\ \text { FJERRU } & >\text { hierro }\end{array}$

Y como dijimos: Es posible que incluso ante [w] como en fuerte, fuente etc. antiguamente se pronunciara $[\mathrm{h}]$ en vez de [f] sin que fuese necesaria la influencia vasca, cf. Penny $(1972: 473)^{30}$ :

However, in some localities, $[\mathrm{h} \phi] \ldots$ was absorbed by $[\mathrm{h}] .[\ldots]$. Movement of $[\mathrm{h} \phi]$ to $[\mathrm{h}]$ is the norm in the other area of preservation of $[\mathrm{h}] \ldots$

Tanto vale para $[\phi]$ ante $[\mathrm{r}]$ y [1] como dijimos más arriba: ${ }^{31}$ Antes de llegar a $[\mathrm{fr}] /[\mathrm{fl}]-$ estadio actual - puede haber existido un estadio intermedio medieval [hr] / [hl], análogo a las condiciones vigentes en gascón.

\section{Resumamos:}

A través de la explicación del fonéma / $\$$ / de Ralph Penny (1972) se puede demostrar que desde el punto de vista combinatorio $[\mathrm{f}]>[\mathrm{h}]$ pudo nacer en cualquier posicion inicial sin necesidad de un factor como el contacto lingüístico. Esto también vale para casos como:

\section{fuerte / huerte (ej. gascón de esta posición: huelha de FOLIA)}

frío / hrio (ej. gascón de esta posición: hret de FRIGDU)

El punto de vista variacional nos muestra que al inicio la innovación $\mathrm{f}->\mathrm{h}$ - no se limitaba a ciertas posiciones iniciales, sino que estaba generalizada como en gascón. Esto significa además que el argumento a.) de Trask no puede ser utilizado para desvalidar la tésis de contacto.

\subsection{Rasgos problemáticos del punto de vista estructural}

Sin embargo, el punto de vista estructuralista presenta algunos problemas que quisiéramos esbozar como sigue:

$\rightarrow$ También en el latín vulgar de Galicia, Asturias, León, Navarra y Aragón el fonéma /f/- sería reemplazado por el fonéma / $\phi$ /

$\rightarrow$ Sin embargo, en las hablas romances de estas zonas no ocurrió el cambio [f]- $>$ [h]-

Penny elimina este dilema en tres etapas, cf. id. (1972: 467):

\footnotetext{
${ }^{30}$ En cuanto al problema de la cronología, véase más arriba, párrafo 2.1 .

${ }^{31}$ Véase arriba, párrafo 2.1 .
} 


\title{
Etapa I.)
}

In most northern varieties of Hispano-Romance, $[\mathrm{h}]$ more or less rapidly reverted to $[\phi]$, to restore greater unity of the phoneme (or $[\phi]$ did not arise in the first place, for similar reasons). This is true of Galician, West and Central Leonese and Aragonese, [...].

Queda la pregunta: ¿Por qué no ocurrió también en el castellano? Una respuesta podría ser: En Castilla existía un factor suplementar que fomentaba [h], cf. Tuten (2003: 132-136), véase abajo.

\section{Etapa II.)}

El hecho de que - contrariamente a lo ocurrido en castellano - en gallego, asturleonés y navarro-aragonés $[\phi]$ se convirtiera en [f] se puede explicar, según Penny (1972: 468), de la manera siguiente:

\begin{abstract}
It seems to me more likely that $[\mathrm{f} \phi]$ and indeed [f] are the result of 'labiodentalization' of primitive $[\phi]$ under the influence of standard castilian (the reason for the appearance of [f] in standard castilian will be dealt with in $\S 10$ ).
\end{abstract}

\section{Etapa III.), cf. Penny (1972: 476ff.):}

El nacimiento de [f]- en castellano ${ }^{32}$ se explica a través de la influencia franco- y occitánicorromance que pudo producirse a partir de finales del siglo XI dada la inmigración transpirenáica de individuos que pertenecían a la nobleza, cf. Penny (1972: 476ff.). Este hecho extralingüístico podría encontrar su análogo en el hecho de que en los préstamos francos, francorromances y árabes tomados antes del siglo XI (es decir antes de la inmigración franco-/occitánicorromance) por los castellano-hablantes, éstos sustituían f- por h-:

(árabe) FÚNDUQ > alhóndiga (,corn-market') etc.

Los préstamos tomados después de la inmigración transpirenáica se impusieron con [f]-:

(fr. ant.) FORGI(E)R $>$ forjar

\section{Resumen de la argumentación de Penny (1972):}

Punto A.)

Por lo que concierne al nacimiento de la innovación f- $>$ h- Penny ofrece una explicación

$\rightarrow$ exclusivamente estructural, o sea, una explicación basada en hechos internos a la lengua (su explicación de 1972 no precisa del contacto lingüístico, en este caso con el vasco, como factor; hemos señalado más arriba los puntos problemáticos).

\footnotetext{
${ }^{32}$ En realidad, el nacimiento de [f]-, precisamente en castellano, parece cosa muy llamativa si consideramos que en esta variedad existía 'como máximo' $[\phi]$ que tendía automáticamente a $[\mathrm{h}]$ (véase más arriba).
} 
Punto B.)

Para explicar la distribución irregular del fenómeno en castellano, Penny sí emplea una explicación basada en el contacto lingüístico, en concreto un factor extralingüístico: Véase más arriba lo referido al franco-/occitánicorromance.

\subsection{Sumando argumentos sociolingüísticos a la discusión estructuralista: La ventaja del planteamiento de Tuten (2003: 132-136)}

Como hemos visto, la argumentación exclusivamente estructural no puede esclarecer todas las dudas. Es aquí donde se muestra la ventaja de las argumentaciones policausales como la de Tuten, ibd., respecto al fenómeno f- $>$ h. En otras palabras: La imagen de "tipping the scales " (en español "dejar el fiel de la balanza/inclinar la balanza") que Tuten (2003: 136) escoge para describir el papel desempeñado por los vascohablantes bilingües podría ser la última tesela del mosaico. En consecuencia, con el planteamiento de Tuten (2003) se puede esclarecer la duda más importante:

¿Si $[\phi]$ existió por todo lado en la Iberorromania septentrional y se convirtió automáticamente en [h], cuál es la causa por la que tan sólo en ciertas zonas de la Iberorromania septentrional se impuso, en muchos casos, esta [h] (nacida de $[\phi]$ )?

La policausalidad de la explicación que nos propone Tuten está basada en el hecho de que principalmente acepta el punto de vista estructural: $[\mathrm{h}]$ sí puede nacer de $[\phi]$ sin necesidad de factores extralingüísticos, sin embargo, emplea argumentos extralingüísticos para demostrar cómo pudo tener lugar la adopción (del resto irregular) de la innovación: Es aquí donde hace falta un factor suplementario, el de la situación de la koinéización en la zona en vía de repoblación:

Los individuos que contribuyeron a la koiné fueron hablantes del

a.) gallego, asturleonés, navarroaragonés, mozárabe (que preferían [f]-)

b.) vascorromance (los vascos que preferían [h]-)

Aunque $\mathrm{f}->\mathrm{h}$ - pudiera haberse originado por factores estructurales, es decir internos a la lengua, según Tuten la interferencia vasca y así el contacto lingüístco pudo haber desempeñado un papel decisivo en lo que concierne a la adopción de la innovación, como dijimos: Según Tuten los vascos bilingües eran "los fieles de la balanza".

Hay otro detalle que distingue el planteamiento de Tuten del de Penny (1972): Tuten (2003: 132-136) parte del principio de que eran los hablantes de gallego, asturleonés, navarroaragonés y mozárabe los que poseían la [f] inicial así que en el "linguistic pool" que utilizaban los hablantes "para formar" 33 la primera koiné castellana se encontraba la [f] inicial, también sin presencia de individuos franco-/occitánicorromances a la que se refiere Penny (1972: 475ss.). Sin embargo, esto no supone problema para la argumentación integral: La manera de Penny, ibd., de explicar el detalle de la re-emergencia de f- en ciertas posiciones (que según él se debe a una influencia franco-/occitánicorromance) esclarece un punto particular del que Tuten (2003: 132-136) no habla en detalle así que no contradice el planteamiento de Tuten, sino que más bien lo completa.

Cabe señalar aquí que en cuanto a la argumentación policausal propuesta por Tuten (2003: 132-136) nos encontramos frente a una analogía respecto a la pregunta de qué papel

\footnotetext{
${ }^{33}$ Que no surjan equivocaciones: Aquí no se hace alusión a un verdadero proceso consciente.
} 
pudo desempeñar el franco como lengua de contacto del galolatín en lo que concierne al nacimiento de la innovación "palatalización de lat. [k] y [g] ante [a]" y su éxito en el galoromance, que así, junto a los demás fenómenos, se convirtió en francorromance, descrita en Jodl (2000) y (2004).

\subsection{El problema de la distribución de f- versus h-}

Según Tuten son los hablantes de gallego, asturleonés, navarroaragonés y mozárabe los que causan una restitución de f- lo que conlleva una distribución irregular de los alófonos. Lo que Tuten no tematiza es cómo se puede explicar que la f- se restituyera precisamente en ciertas posiciones, como "huerte", "huente", "hrío", etc. Dejando, de momento, aparte la explicación que nos dio Penny (1972: 475ss.) con el argumento de la influencia franco/occitánicorromance, se podría dar la respuesta siguiente a la pregunta que acabamos de hacer:

En las demás posiciones como "horno" / "hurto" era - desde el punto de vista físiológico - más fácil que naciera [h], cf. Tuten (2003: 133s.), y desde estas posiciones la innovación podía propagarse a las demás vocales o semi-vocales /a/, /e/, /i/, /j/.

Ante [w], sin embargo, se mantuvo el estadio [h $\phi]^{34}, \mathrm{y}$ ante $[\mathrm{r}]$ y [1] el estadio $[\phi]$, los dos durante más tiempo, ${ }^{35}$ dando lugar, aun en la Edad Media, a $[\mathrm{h}],{ }^{36}$ como "huerte"/"fuerte" o "hrío"37/"frío", porque los inmigrantes franco-/occitánicorromances interpretarían $[h \phi]$ y $[\phi]$ como [f], también por intuición etimológica. En estos casos tardíos, que aun presentaban oscilación fonética, se impuso la [f] - fomentada por la influencia transpirenáica.

Según Tuten, la distribución como la conocemos se debe a la contribución de los hablantes del gallego, asturleonés, navarroaragonés y mozárabe.

\section{La aportación de la sociolingüística histórica}

\subsection{El papel del número de hablantes de una lengua de contacto}

En cuanto al papel que desempeña el mero número de individuos bilingües, es decir de hablantes de una lengua de contacto de la que se presume que ha influenciado la lengua de interés, se ha esgrimido repetidas veces que un influjo no es posible si el número de los individuos alóglotos y bilingües es bajo, cf. Berndt $(1965=1969)^{38}$. Es interesante ver que, sin embargo, varios autores han rechazado esta opinión con los argumentos siguientes, cf. Bailey/Maroldt (1977: 28, a propósito de la cuestión de cómo el frances antiguo influenció el inglés antiguo) a los que nos permitimos citar otra vez (véase arriba):

More important than this parade of numbers [Berndt's requisito de un número bastante alto de individuos expuestos al contacto lingüístico, F.J.], however, seems to be the social status of the immigrants and their language. Berndt is probably right in assuming that the highest nobility [...], whose in-

\footnotetext{
${ }^{34}$ Cf. Penny (1972: 473), véase arriba, 2.1.

${ }^{35} \mathrm{Si}$ no, no habría sido posible que se produjese una restitución por [f].

${ }^{36}$ Esto contradiría Penny (1972: 469, 474ss. y 479ff.) quien es de la opinión de que [hwع] nació tardío, es decir después que el sonido $[\chi]$ naciera de esp. ant. [3] / [ ] . Se puede decir que Penny considera a [hwE] como variante nacida de $[\chi w \varepsilon]$.

${ }^{37}$ Según Penny $(1972: 467+470)$ no está completamente excluido que también el $[\phi]$ en $[\phi r]$ se relajase hacia [hr], véase más arriba 2.1 .

${ }^{38} \mathrm{Cf}$. nota 5 , más arriba.
} 
terests were most intensively engaged in the Continent and who spent most of their time in France, were exclusively French in orientation. This, however, was probably not true of the 6500-7200 knights, the lesser feudal representatives all over the country who were subject to language contact. Intermarriage, English nurses and playmates for the children, and the necessities of everyday life and administration must have caused intensive language contact.

Noll (2009: 82) defiende la misma idea de la intranscendencia del mero número de alóglotos bilingües a propósito de las variedades del español europeo que pueden haber forjado el carácter del español americano:

\begin{abstract}
So können im Prinzip auch wenige Vertreter einer bestimmten Variante hinreichen, um einen Prozess auszulösen, der später zur Generalisierung einer Entwicklung führt. [Así pocos representantes de cierta variante lingüística son suficientes para desencadenar un proceso que luego lleva a la generalización de los rasgos en cuestión. (traducción F.J.)]
\end{abstract}

Sirviéndonos de los progresos que ha hecho la sociolingüística (histórica) podemos descartar el argumento de que el número de hablantes portadores de una innovación es decisivo para su generalización. Esto no quiere decir que incluso un número extremadamente bajo sea capaz de causar generalizaciones sino más bien, que ciertos grupos sociales, aunque no consten de masas de individuos, pueden desempeñar un papel de factor decisivo para el pleno despliegue de una innovación lingüística. ${ }^{39}$ Quisiéramos así centrarnos en los demás factores sociolingüísticos que contribuyen a la formación de una variedad lingüística, en nuestro caso, de una lengua.

\title{
3.2. La distinción del nivel "origen de una innovación" y "generalización de una inno- vación = cambio"
}

Tratemos primero los mecanismos de propagación de innovaciones lingüísticas fijándonos en los siguientes ejemplos:

a.) Inglaterra medieval:

a1.) Nivel de la "innovación lingüística"

Los hablantes del francés antiguo inmigrados en Inglaterra adoptan el inglés antiguo y lo utilizan en la vida cotidiana (cf. arriba citación Bailey/Maroldt) dándole un carácter particular, con nuevos rasgos lingüísticos. Nace así un sociolecto = inglés con innovaciones debidas al contacto con el francés antiguo

a2.) Nivel del "cambio lingüístico"

Este sociolecto o rasgos de él pueden asumir un prestigio tal que logran la generalización. Este proceso aún no explica el nacimiento de la "nueva lengua" en toda su complejidad dado que concurren más factores para formarla, como la influencia de las distintas variedades inglesas o sea: la koineización ${ }^{40}$.

Cum grano salis - este modelo se podría aplicar también a la Castilla proto-medieval para hacer hincapié en las particularidades de la evolución del romance de esta zona.

\footnotetext{
${ }^{39}$ Cf. Jodl (2004) y Jodl (2005) a propósito de un proceso análogo en el francés antiguo y el padanorromance.

${ }^{40}$ Véase también arriba, párrafo 1.1.
} 


\title{
3.2.1. Particularidades del cambio lingüístico en el proto-castellano
}

El criterio social parece desempeñar un papel extraordinariamente importante en la Castilla proto-medieval, lo que nos muestra el hecho de que la innovación $\mathrm{f}>\mathrm{h}$, que hoy día es la norma ${ }^{41}$, haya tenido enormes dificuldades en establecerse para formar parte de ella. Para explicar una evolución tan poco lineal, hay indicios que insinúan que fue el estigma social de la innovación $\mathrm{f}>\mathrm{h}$ la que obstaculó su éxito. Esto lo podemos deducir de ciertos préstamos léxicos, como las palabras francesas honte y hardi, en los que la [h] aun se pronunciaba y que, sin embargo, fueron asumidos por el castellano bajo la forma de fonta y fardido. ${ }^{42}$ Este fenómeno casi no nos deja otra explicación que la de ver en esta particularidad un reflejo de los esfuerzos que la gente culta hacía para combatir la evolución, típica del lenguaje popular, f- $>$ h-. Un caso análogo sería la palabra árabe al-jomra ${ }^{43}$ que ha sido asumida en el vocabulario de los romanófonos bajo las formas "alhombra" y "alfombra", siendo la primera, en concreto "alhombra", la que más se parecía al original "al-jomra": el sonido que sigue el artículo (posición inicial) sería concebido por los romanófonos como [h]. Como dijimos y como demuestra el caso de "la fonta", el sonido [h] debe de haber estado estigmatizado por pertenecer a un registro demasiado bajo. Así se explica que se impusiera la variante con $[\mathrm{f}]$-.

Un apoyo para este planteamiento se recoge en la lingüística variacional, cf. Lapesa (1981: 237):

\begin{abstract}
En la primera mitad del siglo XV pervivían en la lengua muchas inseguridades: no se había llegado a la elección definitiva entre las distintas soluciones que en muchos casos contendían. Así alternaban indiferentemente las grafías $t$ y $d$ finales [...]; la $f$-inicial de fazer, folgar, fuego, preferida por la literatura, luchaba con la [h] aspirada de hazer, holgar, huego, dominantes en el habla; en Castilla la Vieja se extendía la omisión de esta [h] (ebrero 'febrero').
\end{abstract}

Un detalle muy interesante a este respecto son los dobletes como "fallar" y "hallar" y sus dominios sociales de uso, cf. Lapesa $(1981: 313)^{44}$ :

En la primera mitad del siglo XVI se toleraba todavía la $f$ arcaizante de fijo, fincar, fecho, etc.; entre notarios y leguleyos se atestigua todavía a lo largo del siglo XVII. [...]. A este uso cancilleresco debemos la conservación de fallar como término jurídico, al lado del corriente hallar. Otras huellas quedaron de la secular vacilación, como las duplicadas falda y halda, forma y horma. Salvo cultismos muy numerosos - y casos especiales, la $f$ desapareció, sustituida por $h$, que en Castilla la Vieja no se aspiraba ya desde mucho antes. [...]. Por otra parte era propia de la lengua rústica la aspiración [h] en lugar de /f/ ante los diptongos /ue/, /ie/ (huerte, hue, hiebre) y en he 'fe', perheto 'perfecto'.

Como hemos dicho (véase arriba, 2.1.), a esto se suma que - desde el punto de vista variacional - se puede decir que en la primera fase de la innovación $\mathrm{f}$ - $>$ h- no hubo restricción de posición, ni ante [w] ni tampoco ante [r] y [1]. Pero la lingüística variacional no sólo nos

\footnotetext{
${ }^{41}$ Prescindiendo de la distribución un tanto irregular que tratamos arriba.

${ }^{42}$ Cf. Menéndez Pidal (1980: 206) y Lapesa (1981: 127s.) Agradezco al Prof. Dr. Franz-Josef Klein, Siegen, la indicación. La representación más detallada del problema se encuentra en Steiger (1932).

${ }^{43}$ Cf. Alonso (1986: 240, vol. I).

${ }^{44}$ Cf. también el arcaísmo "el fumo" en vez de "el humo" en el español patrimonial del Texas. Agradezco a mi colega Dra. Sandra Herling, Universidad de Siegen, por haberme señalado este detalle. Otro ejemplo, de carácter más general, sería: "fierro" que - en Hispanoamérica - coexiste con "hierro".
} 
permite responder a uno de los cuatro argumentos vertidos por Trask que discutimos más arriba sino que nos lleva también al núcleo, ya insinuado en este párrafo, del presente artículo: errores pueden convertirse en rasgos que forman la norma.

\title{
3.3. El prestigio social de los sociolectos: Un error se vuelve "norma"
}

Integrando los ejemplos de los dobletes aducidos por Lapesa (cf. arriba), quisiéramos tratar aquí, en modo de resumen, un ejemplo de doblete particular por incluir una bifurcación funcional y diastrática, cf. Penny (1972: 478):

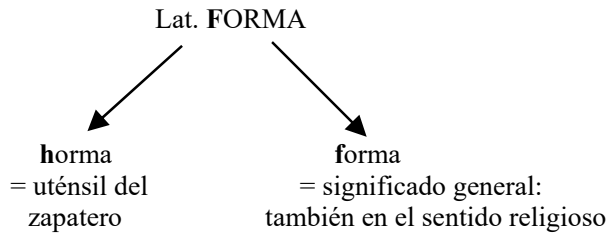

Es aquí donde vemos, una vez más, que la variante con h- al inicio pudo imponerse tan sólo en cierto ámbito social, hecho que causa una distribución muy irregular de la innovación y su asunción a la norma. Si comparamos esta particularidad con la situación en gascón reparamos en el hecho de que en esta variedad la innovación no ha conocido restricciones. ¿Cuales pueden ser las causas de esta diferencia? Ahora bien, la distribución irregular de f$>$ h- se ha explicado, cf. Tuten (2003: 132-136, tratado arriba), a través de la situación de koineización que cundió en la Castilla primitiva. Tal situación no existió en gascón - esta variedad se desarrolló en circunstancias sociales completamente distintas así que la innovación $\mathrm{f}->$ h- causada por interferencia vasca se estableció sin factores suplementares (de koineización). Así se explica su generalización completa, cf. Menéndez Pidal (1980: 221):

\begin{abstract}
La regularidad de la $h$ en gascón se debe, según creo, a que ese dialecto se desarrolló en más tranquilo aislamiento, con más imperturbada peculiaridad, mientras el viejo castellano y el riojano se desarrollaron bajo fuerte presión cultista y en activa comunicación con el leonés y el aragonés-catalán, favorables a la $f$.
\end{abstract}

\section{Conclusión}

De la discusión de los hechos fonéticos resulta que de las dos vertientes de los Pirinéos se desarrolló una variedad lingüística que podría llamarse "bascorromance": el gascón y el castellano, los dos ricos en rasgos de interferencia vasca. Para poder mantener esta manera de ver las cosas, se ha discutido la diferencia de detalle entre el gascón y el castellano referente al fenómeno del rasgo f- $>$ h-. El punto de vista variacional nos proporciona indicios para llegar a la conclusión de que en el castellano más antiguo el carácter de la innovación de $\mathrm{f}->\mathrm{h}$ - $\mathrm{y}$ su distribución básicamente no se distinguía del fenómeno análogo del gascón. Queda claro que es el papel de los hablantes de bascorromance castellano propagar los rasgos lingüísticos de su sociolecto a otras variedades y contribuir a la formación de una koiné y una lengua nacional. Como confirmación de nuestro punto de vista variacionista y para concluir el presente artículo citaremos una vez más al maestro Menéndez Pidal, con las palabras siguientes, cf. id. (1980: 208):

Tenemos que presenciar, no 'una ley fonética' cumplida con exactitud mecánica en el preciso momento de su promulgación, sino una multisecular pugna de formas varias, conviventes, pugna de tenden- 
cias expresivas (llaneza, descuido, purismo, ultracorrección, etc.), y tenemos que ver esas tendencias muy variamente ligadas a través de los siglos con las sucesivas circunstancias históricas, sociales y politicas, del pueblo en que primero se producen y de los pueblos en que después se propagan.

\section{Bibliografía}

Alonso, A. (1986): Diccionario Medieval Español. Desde las Glosas Emilianenses y Silenses (s. X) hasta el siglo XV, vol. 1-2, Salamanca: Universidad Pontificia.

ARgEnTE, Joan A. (1998): "Lenguas en contacto - Sprachen im Kontakt", en: Lexikon der Romanistischen Linguistik, Tübingen: Niemeyer, vol. VII, Art. 458, 1-14.

BAILEY, Charles-James N./Maroldt, Karl. 1977. "The French lineage of English", in: Meisel, Jürgen M. (1977, Hg.): Langues en contact - Pidgins - Creoles - Languages in Contact, Tübingen: Narr, 21-53. (Tübinger Beiträge zur Linguistik Band 75).

BERNDT, Rolf (1965=1969): "The Linguistic Situation in England from the Norman Conquest to the Loss of Normandy (1066-1204)", in: Philologica Pragensia 8, 145-163 [Reprint: Approaches to English Historical Linguistics. An Anthology ed. by Roger Lass, 369-391. New York: Holt, Rinehart and Winston, 1969].

BERTOLDI, Vittorio (1931): "Problèmes de substrat", en: Bulletin de la Société linguistique XXXII, 93-184.

COSERIU, Eugenio (1974): Synchronie, Diachronie und Geschichte. Das Problem des Sprachwandels. München: Fink. (Versión original: Syncronía, diacronía e historia. El problema del cambio lingüístico. Montevideo 1958).

FlORES, L. (1983): Atlas lingüistico y etnográfico de Colombia (ALEC), Bogotá: Instituto Caro y Cuevo.

HoLTUS, Günter/Kramer, Johannes (2010): "Bündnerromanische, dolomitenladinische und friaulische Forschungen 2004-2007/2008”, in: Ladinia 34, 149-185.

JODL, Frank (2000): "Churrätien und das Frankenreich in sprachlicher Hinsicht: Das Zusammenspiel außer- und innersprachlicher Faktoren und mögliche Folgen für die Herausbildung des Bündnerromanischen", in: Annalas da la Societad Retorumantscha $113,109-142$.

JoDL, Frank (2004): Francia, Langobardia und Ascolis Ladinia. Die Bedeutung außersprachlicher Faktoren im Zusammenhang mit innersprachlichen Entwicklungen in drei Teilgebieten der Romania. Frankfurt/Main: Verlag Peter Lang.

JoDL, Frank (2005): 'L'origine della palatalizzazione di $[\mathrm{k}, \mathrm{g} / \mathrm{a}]$ nel romanzo dell'Italia settentrionale, del Ticino, dei Grigioni e della Ladinia dolomitica", in: Ladinia 29, 155 192.

JODL, Frank (2008): "Alles alte Hüte? Die Konzepte Vulgärlatein, Romanisch und kontaktinduzierter Sprachwandel im Lichte der aktuellen Sprachwandeldiskussion", en: Gabriele Blaikner-Hohenwart u.a. (Hgg.): Ladinometria. Festschrift für Hans Goebl zum 65. Geburtstag, 2 Bände, Salzburg/Bozen/Vich (TN)/San Martin de Tor (BZ): Universität Salzburg - Fachbereich Romanistik/ Freie Universität Bozen/Istitut Cultural Ladin "majon di fascegn" (Vich)/Istitut Ladin "Micurà de Rü“ (San Martin de Tor), Band 2, 159-178.

JODL, Frank (2013a): "Mille ans de peu d'importance? Les éthno-préfixes «gallo»- et «franco»- et le message de leur usage : mono- versus polycausalité", en : Revista de Filología Románica 30/1, 37-52.

JODL, Frank (2013b): "Spanisch in Nicaragua", in: Herling, Sandra/Patzelt, Carolin (eds.): Weltsprache Spanisch: Variation, Soziolinguistik und geographische Verbreitung des 
Spanischen. Handbuch für das Studium der Hispanistik. Stuttgart: Ibidem, 593-617.

JoDL, Frank (2013c, en prep.): "Fisiología y cognición - el cambio fonético y las teorías acerca del cambio lingüístico más recientes: el cambio [f]- > [h]- en castellano antiguo", en: Fernández Poza, Óscar/Muñoz Carrobles, Diego (Hgg.): Filología Románica Hoy.

JoDL, Frank (2016): "Guerra del Castellano und Convivencia: Die Aktualität des Mittelalters für die akademische Lehre", en: Hispanorama 152, 94-105.

KABATEK, Johannes (2005): "Über Trampelpfade, sichtbare Hände und Sprachwandelprozesse", en Stehl, Thomas (ed.): Unsichtbare Hand und Sprecherwahl. Typologie und Prozesse des Sprachwandels in der Romania, Tübingen: Narr, 155-174.

KEller, Rudi (1994): Sprachwandel: Von der unsichtbaren Hand in der Sprache, Tübingen/Basel: Francke.

$\mathrm{KoCH}$, Peter (2005): "Sprachwandel und Sprachvariation", in: Schrott, Angela/Völker, Harald (eds.): Historische Pragmatik und historische Varietätenlinguistik in den romanischen Sprachen. Göttingen: Univ.-Verlag, 229-254.

LABOV, William (1994): Principles of linguistic change. Volume 1: Internal factors. Oxford: Blackwell.

LABOV, William (2001): Principles of linguistic change. Volume 2: Social factors. Oxford: Blackwell.

LAPESA, Rafael (2008/1981): Historia de la lengua española. Madrid: Gredos.

LASS, Roger (1997): Historical linguistics and language change. New York: Cambridge University Press. (Cambridge Studies in Linguistics 81).

Lloyd, Paul M. (1987): From Latin to Spanish. Philadelphia: American Philosophical Society.

LÜDTKE, Helmut (1980): "Sprachwandel als universales Phänomen", en: Helmut Lüdtke (ed.). Kommunikationstheoretische Grundlagen des Sprachwandels. Berlin/New York: De Gruyter. 1-19.

LÜDTKE, Helmut (1986): "Equisse d'une Théorie du Changement langagier", en: La Linguistique 22 (fasc. 1), 3-46.

MARTINET, André (1951-52): “The unvoicing of Old Spanish sibilants", in: Romance Philology 5, 141-145.

MenÉndez PIDAL, Ramón $\left({ }^{5} 1964 /{ }^{9} 1980\right)$ : Orígenes del español. Estado lingüistico de la Península Ibérica hasta el siglo XI. Madrid: Espasa Calpe.

NARO, Anthony (1972a): "On 'f- > h-' in Castilian and Western Romance", in: Zeitschrift für Romanische Philologie 88, 435-447.

NARO, Anthony (1972b): “A reply", in: Zeitschrift für Romanische Philologie 88, 459-462.

Navarro, Tomás (1974): El español de Puerto Rico, San Juan: Universidad de Puerto Rico - Río Piedras.

NEZIROVIC, Muhamed (2002): “Judenspanisch”, en: OKUKA 2002, 101-116.

NoLl, Volker (2009): Das amerikanische Spanisch. Ein regionaler und historischer Überblick. 2., aktualisierte und erweiterte Auflage. Tübingen: Niemeyer.

PENNY, Ralph (1972): “The reemergence of /f/ as a phoneme in Castilian”, en: Zeitschrift für Romanische Philologie 88, 463-482.

PENNY, Ralph (1991): A history of the Spanish language, Cambride: Cambride University Press.

PENNY, Ralph (2009): "La pertinencia de la dialectología y de la sociolingüística para la historia del español: Unidad y divergencia en el romance peninsular", in: Laura Romero 
Aguilera \& Carolina Julià Luna. Hgg. Tendencias actuales en la investigación diacrónica de la lengua: Aactas del VIII congreso nacional de la Asociación de Jóvenes Investigadores de Historiografia e Historia de la Lengua Española (AJIHLE), Barcelona, del 2 al 4 de abril de 2008. Barcelona: Publicacions i Edicions de la Universitat de Barcelona, 45-55.

Quesada Pacheco, Miguel Angel. 2002/2008. El español de América, Cartago/Costa Rica: Editorial Tecnológica de Costa Rica. Erstauflage 2000, Neudruck 2008.

QuILIS, Antonio. 1995. „Notas de fonética del español de Nicaragua“, in: Blesa, Túa/Martín Zorraquino, María Antonia (Hgg.): Homenaje a Félix Monge, Madrid: Gredos.

Ramos Remedios, Emilia (2000): Los cartularios de Santa María de Valpuesta. Análisis lingüístico, Donostia/San Sebastián: Eusko Ikaskuntza. (Colleción Lankidetzan 17).

Rivarola, José Luis (1972): "Sobre $\mathrm{f}>\mathrm{h}$ en español", in: Zeitschrift für Romanische Philologie 88, 448-458.

Rosales Solís, María Auxiliodora. 2010. "El español de Nicaragua“, in: Quesada Pacheco, Miguel Angel (Hg.): El español hablado en América Central. Madrid/Frankfurt a.M.: Iberoamericana/Vervuert, 137-154.

Rueda Chaves, John E./Navas Escorcia, Lester M. (2011): "Variación sociofonológica en la fricativa labiodental sorda /f/ en el español hablado en Barranquilla", en: Cuadernos de Lingüistica/U.P.R.Working Papers, vol.3 no.1, 39- 63.

Online:

http://linguistica.uprrp.edu/Articulos\%20septiembre\%202011/Variacion $\% 20$ sociofonolo gica $\% 20$ en $\% 201 \mathrm{a} \% 20$ fricativa $\% 201$ abiodental $\% 20$ sorda.pdf

fecha de consulta: 1 de diciembre 2016

SCHUCHARDT, Hugo (1866/1975): Der Vokalismus des Vulgärlateins. Hildesheim: Olms [Reimpresión de la edición de Leipzig, $\left.{ }^{1} 1866-1868\right]$.

STEIGER, Arnald (1932): Contribución a la fonética del hispano-árabe y de los arabismos en el ibero-románico y en el siciliano, Madrid: Ed. Hernando. (Revista de Filología española, Anejo 17).

TRASK, Robert L. (1997a): The History of Basque. London: Routledge.

TRASK, Robert L. (1997b): A Student's Dictionary of Language and Linguistics. London: Hodder Arnold.

TrudgiLl, Peter (1986): Dialects in Contact. Oxford: Blackwell.

TRUDGILL, Peter (2008a): "Colonial dialect contact in the history of European languages: On the irrelevance of identity to new-dialect formation", in: Language in Society 37, 241-254.

TRUDGiLl, Peter (2008b): "On the role of children, and the mechanical view: A rejoinder", in: Language in Society 37, 277-280.

Tuten, Donald N. (2003): Koineization in medieval Spanish. Berlin [u.a.]: Mouton de Gruyter.

TUTEN, Donald N. (2008): „Identity formation and accomodation: Sequential and simultaneous relations", in: Language in Society 37, 259-262.

WARTBURG, Walther von (1928): Recensión de: Menéndez Pidal, Ramón (1926): Orígenes del español. Estado lingüístico de la Península Ibérica hasta el siglo XI. Revista de Filolgía Románica; Anejo 1, Madrid, 1ª edición”, en: Zeitschrift für romanische Philologie XLVIII, 457-461. 